International Journal of Pure and Applied Mathematics

Volume 92 No. 4 2014, 481-497

ISSN: 1311-8080 (printed version); ISSN: 1314-3395 (on-line version)

url: http://www.ijpam.eu

doi: http://dx.doi.org/10.12732/ijpam.v92i4.4

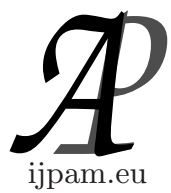

\title{
SOME SUZUKI TYPE FIXED POINT THEOREMS FOR MULTI-VALUED MAPPINGS AND APPLICATIONS
}

\author{
Ramendra Krishna Bose \\ Department of Mathemtics \\ University of Texas-PanAmerican \\ Edinburg, Texas 78539, USA
}

\begin{abstract}
Recently Suzuki (2008) and then Kikkawa and Suzuki (2008) gave a new generalization of the Banach contraction principle. Then, Mot and Petrusel (2009), Dhompapangasa and Yingtaweesttikulue (2009), Bose and Roychowdhury (2011), Singh and Mishra (2010), and Doric and Lazovic (2011) further extended their work. Recently Bose (2012) obtained some Suzuki-type common fixed point theorems for generalized contractive multivalued mappings using a result of Bose and Mukherjee(1977) which extend the previously obtained results.
\end{abstract}

Recently Damjanovic and Doric(2011) obtained a multivalued generalization of theorems Kikkawa and Suzuki (2008) concerning Kannan mappings. Also Singh and Mishra (2010) considered coincidence and fixed point theorems for a class of hybrid pair of single-valued and multi-valued maps in metric space setting and Singh et al (2012) prsented a common fixed point theorem for a pair of multi-valued maps in a complete metric space extending a recent theorem of Doric and Lazovic. First we generalize the theorem of Singh et al which also extend the theorem of Singh and Mishra(2010). Then we extend the theorem of Damjanovic and Doric to a common fixed point theorem of a pair of multivalued mappings. As an application, we consider the existence of a common solution for a class of functional equations arising in dynamic programming.

AMS Subject Classification: 47H10, 47H04, 54H25

Received: August 3, 2013

(c) 2014 Academic Publications, Ltd. url: www.acadpubl.eu 
Key Words: common fixed point, coincidence point, contractive multifunction, Hausdorff-Pompeiu metric

\section{Introduction}

The Banach contraction principle plays a very impotant role in nonlinear analysis and has several generalizations([12] and the references there in). Recently, Suzuki [19] gave a new type of generalization of the Banach contraction principle. Then Kikkawa and Suzuki [12] gave another generalization which generalized the work of Suzuki and the Nadler fixed point theorem. Very recently Bose and Roychowdhury [3] presented a theorem concerning $(\theta, L)$-multivalued weak contraction which generalized the work of Kikkawa and Suzuki, Nadler [15], Kamran [11], and Berinde and Berinde [1]. Also Mot and Petrusel [14] gave another generalization concerning special multivalued generalized contractions which extended the result of Kikkawa and Suzuki. Singh and Mishra[16] also obtained a similar type theorem for a generalized contraction. In Bose [4], some Kikkawa-Suzuku type theorems concerning the generalized multivalued contractions were presented which extends the work of Singh and Mishra [16], Bose and Roychowdhury [3], Mot and Petrusel [14], Kikkawa and Suzuki [12], and others, a new coincidence point theorem concerning a hybrid pair of mappings $f: X \rightarrow X$ and $T: X \rightarrow C B(X)$ was also discussed and an application concerning dynamic programming was discussed.

Recently Damjanovic and Doric(2011,[8]) obtained a multi-valued generalization of theorems Kikkawa and Suzuki (2008, [13]) concerning Kannan mappings. Also Singh and Mishra (2010, [17]) considered coincidence and fixed point theorems for a class of hybrid contraction consisting of a pair of singlevalued and multi-valued maps in metric space setting and Singh et al (2012, [18]) prsented a common fixed pont theorem for a pair of multi-valued maps in a complete metric space extending a recent theorem of Doric and Lazovic[10]. These are all Suzuki-type theorems. Here we extend the theorem of Damjanovic and Doric [8] to a common fixed point theorem of a pair of multivalued mappings. Also we generalize the theorem of Singh et al[18] which also extend the theorem of Singh and Mishra [17].

\section{Peliminaries}

Let $(X, d)$ be a metric space and let $H$ represents the Hausdorff-Pompeiu metric induced by the metric $d$. Let $C B(X)$ denotes the set of nonempty closed and 
bounded subsets of $X, K(X)$ be the set of nonempty compact subsets of $X$ and $C L(X)$ be the family of nonempty closed subsets of $X$.

$H(A, B)=\max \{\rho(A, B), \rho(B, A)\}$ where $\rho(A, B)=\sup _{a \in A} D(a, B)$

and $D(a, B)=\inf _{b \in B} d(a, b)$ (Some time $d(a, B)$ instead of $D(a, B)$ ).

Note that $(C B(X), H)$ is a complete metric space when $(X, d)$ is a complete metric space. Also $(C L(X), H)$ is a complete generalized metric space( in the sense of Luxemburg-Jung, Covitz\& Nadler Jr.[7]).

Theorem A. (see Kikkawa and Suzuki [12]) Let $(X, d)$ be a complete metric space and $F: X \rightarrow C B(X)$ a multivalued mapping. Assume that there exists $r \in[0,1)$ such that $\frac{1}{1+r} D(x, F x) \leq d(x, y)$ implies that $H(F x, F y) \leq r d(x, y)$ for all $x, y \in X$. Then $F$ has a fixed pont.

Theorem B. (see Suzuki [19]) For a metric space $(X, d), X$ is complete if and only if every mapping $T$ on $X$ such that there exists $r \in[0,1)$, $\theta(r) D(x, T x) \leq d(x, y)$ implies that $d(T x, T y) \leq r d(x, y$ for all $x, y \in X$, has a fixed point where $\theta:[0,1) \rightarrow\left(\frac{1}{2}, 1\right]$ is a onto and nonincreasing function defined below:

$$
\theta(r)= \begin{cases}1, & 0 \leq r \leq \frac{\sqrt{5}-1}{2} \\ (1-r) r^{-2}, & \frac{\sqrt{5}-1}{2} \leq r<2^{-\frac{1}{2}} \\ (1+r)^{-1}, & 2^{-\frac{1}{2}} \leq r<1\end{cases}
$$

Later, Mot and Petrusel extended the Theorm A as follows:

Theorem C. (see Mot and Petrusel [14]) Let $(X, d)$ be a complete metric space and $F: X \rightarrow C L(X)$ a multifunction. Assume that there exist $\alpha . \beta, \gamma \in$ $[0,1)$ such that $\alpha+\beta+\gamma<1$ and $\frac{1-\beta-\gamma}{1+\alpha} D(x, F x) \leq d(x, y)$ implies that $H(F x, F y) \leq \alpha d(x, y)+\beta D(x, F x)+\gamma D(y, F y)$ for all $x, y \in X$. Then $F$ has a fixed point.

Definition 2.1. Let $(X, d)$ be a metric space and let $F: X \rightarrow C(X) / C B(X)$ a muti-valued operator. $F$ is said to be a multi-valued weakly Picard operator ( briefly MWP operator) if for all $x \in X$ and all $y \in F x$, there exists a sequence $\left\{x_{n}\right\}$ such that $x_{n+1} \in F x_{n}$, for all for all $n=0,1,2,3 \ldots$ starting with any $x_{0} \in X$ and the sequence is convergent and its limit is a fixed point of $F$.

Definition 2.2. (see [3]) Let $(X, d)$ be a metric space, $f: X \rightarrow X$ be a self-mapping and $T: X \rightarrow C B(X)$ be a multi-valued mapping. The mapping $f$ 
and $T$ are called $R$-weakly commuting if for given $x \in X, f(T(x)) \in C B(X)$ and there exists some real number $R$ such that

$$
H(f(T(x)), T(f(x))) \leq R d(f(x), T(x)) .
$$

Definition 2.3. (see [3]) The mappings $f: X \rightarrow X$ and $T: X \rightarrow C B(X)$ are weakly compatible if they commute at their coincidence points, i.e., if $f(T(x))=T(f(x))$ whenever $f(x) \in T(x)$.

Theorem D. (see Bose and Roychowdhury [3], Theorem 3.2) Let $(X, d)$ be a complete metric space and $T: X \rightarrow C B(X)$ a multivalued mapping and $f: X \rightarrow X$. Suppose that there exist two constants $\theta \in[0.1)$ and $L \geq 0$ such that

$$
\begin{aligned}
& \eta(\theta) d(f(x), T x) \leq d(f(x), f(y)) \\
& \Longrightarrow H(T x, T y) \leq \theta d(f(x), f(y)))+L D(f(y), T x),
\end{aligned}
$$

for all $x, y \in X$, where $\eta:[0,1) \rightarrow\left(\frac{1}{2+L}, \frac{1}{1+L}\right)$ defined by $\eta(\theta)=\frac{1}{1+\theta+L}$ is a strictly decreasing function, $T(X) \subset f(X)$ and $f(X)$ is complete. Then

(i) the set of coincidence point of $f$ and $T$ is non-empty, that is, there exists $u \in X$ such that $f(u) \in T u$.

(ii) for any $x_{0} \in X$, there exists an $f$-orbit $O_{f}\left(x_{0}\right)=\left\{f\left(x_{n}\right): n=0,1,2\right.$, $3, \ldots\}$ of $T$ at the point $x_{0}$ such that $f\left(x_{n}\right) \rightarrow f(u)$, where $u$ is a coincidence point of $f$ and $T$, for which the following estimates hold:

$$
\begin{aligned}
& d\left(f\left(x_{n}\right), f(u)\right) \leq \frac{h^{n}}{1-h} d\left(f\left(x_{0}\right), f\left(x_{1}\right)\right), \quad n=0,1,2,3, \ldots \\
& d\left(f\left(x_{n}\right), f(u)\right) \leq \frac{h}{1-h} d\left(f\left(x_{n-1}\right), f\left(x_{n}\right)\right), \quad n=0,1,2,3, \ldots
\end{aligned}
$$

for a certain constant $h<1$. Further, if $f$ and $T$ are $R$-weakly commuting at $u$ and $f(f(u))=f(u)$, then $f$ and $T$ have a common fixed point.

Lemma 2.4. (see Nadler [15]) If $A, B \in C B(X)$ and $x \in A$, then for any positive number $\alpha$ there exists $y \in B$ such that $d(x, y) \leq H(A, B)+\alpha$, that is, $d(x, y) \leq q H(A, B)$ where $q>1$.

Theorem E. (see Bose [4]) Let $(X, d)$ be a complete metric space. Let $C B(X)$ denote the space of nonempty closed bounded subsets of $X$ equipped 
with the Hausdorff metric $H$. Let $F$ and $G$ be a pair of mappings from $X$ into $C B(X)$ satisfying the following conditions:

$$
\begin{aligned}
& \alpha \min \{D(x, F x), D(y, G y)\} \leq d(x, y) \\
& \Longrightarrow H(F x, G y) \leq a_{1} D(x, F x)+a_{2} D(y, G y) \\
& \quad+a_{3} D(y, F x)+a_{4} D(x, G y)+a_{5} d(x, y)
\end{aligned}
$$

for all $x, y \in X$, where $a_{i}, i=1,2,3,4,5$ are non-negative numbers and $\sum_{0}^{5} a_{i}<$ 1 and $a_{1}=a_{2}$ or $a_{3}=a_{4}$. Here $\alpha=\min \{\gamma, \delta\}$, where

$$
\gamma=\frac{1-a_{1}-a_{3}}{1-a_{3}+a_{4}+a_{5}} \quad \text { and } \quad \delta=\frac{1-a_{2}-a_{4}}{1+a_{3}-a_{4}+a_{5}} .
$$

Then there exists a point $u \in X$ such that $u \in F(u)$ and $u \in G(u)$, i.e., $u$ is a common fixed point of $F$ and $G$.

Recently Doric and Lazovic extended and generalized the fixed point theorems of Ciric, Kikkawa and Suzuki and others in the following theorem:

Theorem F. (see Doric and Lazovic [10]) Let $\phi$ be non-increasing function from $[0,1)$ onto $(0,1]$ defined by

$$
\phi(r)= \begin{cases}1, & 0 \leq r<\frac{1}{2}, \\ (1-r), & \frac{1}{2} \leq r<1 .\end{cases}
$$

Let $(X, d)$ be a complete metric space and let $T: X \rightarrow C B(X)$. Assume that there exists $r \in[0,1)$ such that for every $x, y \in X$,

$$
\phi(r) d(x, T x) \leq d(x, y)
$$

implies

$$
H(T x, T y) \leq r \cdot \max \left\{d(x, y), d(x, T x), d(y, T y), \frac{d(x, T y)+d(y, T x)}{2}\right\} .
$$

Then there exists $z \in X$ such that $z \in T z$.

Theorem G. (see Ciric [5], also refer to[6]) Let $(X, d)$ be a complete metric space and let $S, T: X \rightarrow X$. Assume there exists $r \in[0,1)$ such that

$$
d(S x, T y) \leq r \cdot \max \left\{d(x, y), d(x, S x), d(y, T y), \frac{d(x, T y)+d(y, S x)}{2}\right\} .
$$


for all $x, y \in X$. Then $S$ and $T$ have a unique common fixed point.

Theorem H. (see S.L. Singh et al [18]) Let $(X, d)$ be a complete metric space and let $S, T: X \rightarrow C L(X)$. Assume that there exists $r \in[0,1)$ such that for every $x, y \in X$,

$$
\phi(r) \min \{d(x, S x), d(y, T y)\} \leq d(x, y)
$$

where $\phi(r)$ is defined in Theorem $F$, implies

$$
H(S x, T y) \leq r \cdot \max \left\{d(x, y), d(x, S x), d(y, T y), \frac{d(x, T y)+d(y, S x)}{2}\right\} .
$$

for all $x, y \in X$.

Then there exists $z \in X$ such that $z \in S z \cap T z$.

Definition 2.5. (Orbitally Complete) An orbit of $T: X \rightarrow C L(X)$ at $x_{0} \in$ $X$ is a sequence $\left\{x_{n} \in T x_{n-1}\right\}, n=1,2,3, \ldots$. A space $X$ is called $T$-orbitally complete if and if only every Cauchy sequence of the form $\left\{x_{n_{i}} \in T x_{n_{i}-1}\right\}$, $i=1,2, \ldots$ converges in $X$.

S.L. Singh and S. N. Mishra [17] proved the following result (see Corollary 3.2 in $[17])$.

Theorem I. (see Singh and Mishra [17]) Let $(X, d)$ be a complete metric space and let $T: X \rightarrow C L(X)$. Assume that there exists $r \in[0,1)$ such that for every $x, y \in X$,

$$
\eta(r) d(x, T x) \leq d(x, y)
$$

where $\eta:[0,1) \rightarrow\left(\frac{1}{2}, 1\right]$ defined by $\eta(r)=\frac{1}{1+r}$, implies

$$
H(T x, T y) \leq r \cdot \max \left\{d(x, y), \frac{d(x, T x)+d(y, T y)}{2}, \frac{d(x, T y)+d(y, T x)}{2}\right\} .
$$

for all $x, y \in X$.

If there exists a $u_{0} \in X$ such that $X$ is $\left(T, u_{0}\right)$-orbitally complete, then $T$ has a fixed point.

Definition 2.6. Let $(X, d)$ be a complete space and let $T: X \rightarrow X$. Then, $T$ is called a Kannan mapping if there exists $\alpha \in\left[0, \frac{1}{2}\right)$ such that

$$
d(T x, T y) \leq \alpha d(x, T x)+\alpha d(y, T y)
$$


for all $x, y \in X$.

If $T: X \rightarrow X$ satisfies

$$
d(T x, T y) \leq r \cdot \max \{d(x, T x), d(y, T y)\}
$$

for all $x, y \in X$ for some $r \in[0,1)$, then $T$ is called a generalized Kannan mapping.

Theorem J. (see Kikkawa and Suzuki [13]) Let $(X, d)$ be a complete metric space and let $T: X \rightarrow X$ and let $\psi$ be a non-increasing function from $[0,1)$ onto $\left(\frac{1}{2}, 1\right]$ defined by

$$
\psi(r)=\left\{\begin{array}{cc}
1, & 0 \leq r<\frac{1}{\sqrt{2}} \\
\frac{1}{1+r}, & \frac{1}{\sqrt{2}} \leq r<1
\end{array}\right.
$$

Let $\alpha \in\left[0, \frac{1}{2}\right)$ and put $r=\frac{\alpha}{1-\alpha} \in\left[0, \frac{1}{2}\right)$. Suppose that

$$
\psi(r) d(x, T x) \leq d(x, y)
$$

implies

$$
d(T x, T y) \leq \alpha d(x, T x)+\alpha d(y, T y)
$$

for all $x, y \in X$. Then $T$ has a unique fixed point $z$ and $\lim _{n \rightarrow \infty} T^{n} x=z$ holds for every $x \in X$.

Theorem K. (see Kikkawa and Suzuki [13]) Let $(X, d)$ be a complete metric space and let $T: X \rightarrow X$. Let $\theta:[0,1) \rightarrow\left(\frac{1}{2}, 1\right]$ is a onto and non-increasing function defined below:

$$
\theta(r)=\left\{\begin{array}{l}
1,0 \leq r \leq \frac{\sqrt{5}-1}{2} \\
(1-r) r^{-2}, \frac{\sqrt{5}-1}{2} \leq r<2^{-\frac{1}{2}} \\
(1+r)^{-1}, 2^{-\frac{1}{2}} \leq r<1 .
\end{array}\right.
$$

Assume that there exists $r \in[0,1)$ such that for every $x, y \in X$,

$$
\theta(r) d(x, T x) \leq d(x, y)
$$

implies

$$
d(T x, T y) \leq r \cdot \max \{d(x, T x), d(y, T y)\}
$$


Then $T$ has a unique fixed point $z$ and $\lim _{n \rightarrow \infty} T^{n} x=z$ holds for every $x \in X$.

Damjanovic and Doric proved recently the following multi-valued extension of the above theorem.

Theorem L. (see Damjanovic and Doric [8]) Let $\phi$ be nonincreasing function from $[0,1)$ onto $(0,1]$ defined by

$$
\varphi(r)=\left\{\begin{array}{l}
1, \quad 0 \leq r<\frac{\sqrt{5}-1}{2} \\
(1-r), \frac{\sqrt{5}-1}{2} \leq r<1
\end{array}\right.
$$

Let $(X, d)$ be a complete metric space and let $T: X \rightarrow C B(X)$. Assume that there exists $r \in[0,1)$ such that for every $x, y \in X, \phi(r) d(x, T x) \leq d(x, y)$ implies

$$
H(T x, T y) \leq r \cdot \max \{d(x, T x), d(y, T y)\}
$$

Then there exists $z \in X$ such that $z \in T z$.

Definition 2.7. Let $\Phi:[0 . \infty)^{5} \rightarrow[0, \infty)$ be continuous(or upper semicontinuous) and incrasing in each coordinate variable and $\Phi(t, t, t, a t, b t) \leq t$ for every $t \in[0 . \infty)$, where $a+b=2, a, b \in\{0,1,2\}$.

\section{Main Theorems}

Theorem 3.1. Let $(X, d))$ be a complete metric space and let $S, T: X \rightarrow$ $C L(X)$. Assume that there exists $r \in[0,1)$ such that for every $x, y \in X$,

$$
\phi(r) \min \{D(x, S x), D(y, T y)\} \leq d(x, y),
$$

where

$$
\phi(r)=\left\{\begin{array}{l}
1, \quad 0 \leq r<\frac{1}{2} \\
(1-r), \frac{1}{2} \leq r<1
\end{array}\right.
$$

implies

$$
H(S x, T y) \leq r \Phi\{d(x, y), D(x, S x), D(y, T y), D(x, T y), D(y, S x)\} .
$$


Then there exists a $u \in X$ such that $u \in S u \cap T u$ (Here $\Phi$ is as specified in Definition 2.7).

Proof. Starting with $u_{0} \in X$, define a sequence such that

$$
u_{2 n+1} \in T u_{2 n} \quad \text { and } \quad u_{2 n+2} \in S u_{2 n+1}
$$

using Lemma 2.4.

Then in either case, we have

$$
\phi(r) \min \left\{d\left(u_{2 n-1}, S u_{2 n-1}\right), d\left(u_{2 n}, T u_{2 n}\right)\right\} \leq d\left(u_{2 n-1}, u_{2 n}\right)
$$

This implies

$$
\begin{array}{r}
H\left(S u_{2 n-1}, T u_{2 n}\right) \leq r \Phi\left\{d\left(u_{2 n-1}, u_{2 n}\right), D\left(u_{2 n-1}, S u_{2 n-1}\right), D\left(u_{2 n}, T u_{2 n}\right),\right. \\
\left.D\left(u_{2 n-1}, T u_{2 n}\right), D\left(u_{2 n}, S u_{2 n-1}\right)\right\} .
\end{array}
$$

Then

$$
\begin{aligned}
d\left(u_{2 n}, u_{2 n+1}\right) \leq & k H\left(S u_{2 n-1} T u_{2 n}\right), \quad k>1 \\
\leq & \beta \Phi\left\{d\left(u_{2 n-1}, u_{2 n}\right), D\left(u_{2 n-1}, S u_{2 n-1}\right),\right. \\
& \left.D\left(u_{2 n}, T u_{2 n}\right), D\left(u_{2 n-1}, T u_{2 n}\right), D\left(u_{2 n}, S u_{2 n-1}\right)\right\} \quad \beta=r k<1 \\
\leq & \beta \Phi\left\{d\left(u_{2 n-1}, u_{2 n}\right), d\left(u_{2 n-1}, u_{2 n}\right), d\left(u_{2 n}, u_{2 n+1}\right), d\left(u_{2 n-1}, u_{2 n+1}\right), 0\right\} \\
\leq & \beta \Phi\left\{d\left(u_{2 n-1}, u_{2 n}\right), d\left(u_{2 n-1}, u_{2 n}\right), d\left(u_{2 n}, u_{2 n+1}\right), d\left(u_{2 n-1}, u_{2 n}\right)\right. \\
& \left.+d\left(u_{2 n}, u_{2 n+1}\right), 0\right\} .
\end{aligned}
$$

If $d\left(u_{2 n}, u_{2 n+1}\right)>d\left(u_{2 n-1}, u_{2 n}\right)$, we arrive at a contradiction. Hence

$$
d\left(u_{2 n}, u_{2 n+1}\right) \leq d\left(u_{2 n-1}, u_{2 n}\right)
$$

and this leads to

$$
\begin{gathered}
d\left(u_{2 n}, u_{2 n+1}\right) \leq \beta \Phi\left\{d\left(u_{2 n-1}, u_{2 n}\right), d\left(u_{2 n-1}, u_{2 n}\right), d\left(u_{2 n-1}, u_{2 n}\right),\right. \\
\left.2 d\left(u_{2 n-1}, u_{2 n}\right), 0\right\} \leq \beta d\left(u_{2 n-1}, u_{2 n}\right) .
\end{gathered}
$$

That is, $d\left(u_{2 n}, u_{2 n+1}\right) \leq \beta d\left(u_{2 n-1}, u_{2 n}\right)$. In similar manner, we have

$$
d\left(u_{2 n+2}, u_{2 n+1}\right) \leq \beta d\left(u_{2 n+1}, u_{2 n}\right) .
$$

From this, we have $d\left(u_{n+1}, u_{n}\right) \leq \beta d\left(u_{n}, u_{n-1}\right), n \in N$. Therefore the sequence $\left\{u_{n}\right\}$ is a Cauchy sequence and has a limit $u \in X$. 
Next we prove that for any $y \in X-\{u\}$,

$$
\begin{aligned}
& D(u, T y)) \leq r \cdot \max \{d(u, y), D(y, T y)\}, \\
& D(u, S y)) \leq r \cdot \max \{d(u, y), D(y, S y)\} .
\end{aligned}
$$

Since the sequence $\left\{u_{n}\right\}$ converges to $u$, there exists $n_{0} \in N$ such that $d\left(u, u_{n}\right) \leq \frac{1}{3} d(u, y)$ for $y \neq u$ and for all $n \geq n_{0}$. We have

$$
\begin{aligned}
\phi(r) D & \left(u_{2 n-1}, S u_{2 n-1}\right) \leq d\left(u_{2 n-1}, u_{2 n}\right) \leq d\left(u_{2 n-1}, u\right)+d\left(u, u_{2 n}\right) \\
& \leq \frac{2}{3} d(y, u)=d(y, u)-\frac{1}{3} d(y, u) \leq d(y, u)-d\left(u_{2 n-1}, u\right) \leq d\left(u_{2 n-1}, y\right) .
\end{aligned}
$$

Now either $D\left(u_{2 n-1}, S u_{2 n-1}\right) \leq D(y, T y)$ or $D(y, T y) \leq D\left(u_{2 n-1}, S u_{2 n-1}\right)$. In either case, we have

$$
\phi(r) \min \left\{D\left(u_{2 n-1}, S u_{2 n-1}\right), D(y, T y)\right\} \leq d\left(u_{2 n-1}, y\right)
$$

Also we have

$$
\begin{aligned}
D\left(u_{2 n}, T y\right) \leq & H\left(S u_{2 n-1}, T y\right) \\
\leq & r \Phi\left\{d\left(u_{2 n-1}, y\right), D\left(u_{2 n-1}, S u_{2 n-1}\right), D(y, T y),\right. \\
& \left.D\left(u_{2 n-1}, T y\right), D\left(y, S u_{2 n-1}\right)\right\} \\
\leq & r \Phi\left\{d\left(u_{2 n-1}, y\right), d\left(u_{2 n-1}, u_{2 n}\right), D(y, T y), D\left(u_{2 n-1}, T y\right), d\left(y, u_{2 n}\right)\right\} .
\end{aligned}
$$

Taking limit as $n \rightarrow \infty$, we have

$$
d(u, T y) \leq r \Phi\{d(u, y), 0, D(y, T y), D(u, T y), d(y, u)\}
$$

This implies that

$$
d(u, T y) \leq r d(u, y) \quad \text { or } \quad d(u, T y) \leq r d(y, T y)
$$

that is, $D(u, T y) \leq r \cdot \max \{d(u, y), d(y, T y)\}$. Similarly it can be shown that

$$
D(u, S y) \leq r \cdot \max \{d(u, y), d(y, S y)\}
$$

Now we prove that $u \in S u \cap T u$.

Let $0 \leq r<\frac{1}{2}$. Then following three cases are to be considered.

Case I. Suppose $u \notin S u$ and $u \notin T u$.

Let $a \in T u$ be such that $2 r d(a, u)<D(u, T u)$. We have also

$$
D(u, T a)) \leq r \cdot \max \{d(u, a), D(a, T a)\}
$$




$$
D(u, S a)) \leq r \cdot \max \{d(u, a), D(a, S a)\}
$$

Since $\phi(r) D(u, T u) \leq D(u, T u) \leq d(u, a)$, we have $\phi(r)\{D(a, S a), D(u, T u)\}$ $\leq d(u, a)$. This implies

$$
\begin{aligned}
D(S a, a) & \leq H(S a, T u) \\
& \leq r . \Phi\{d(a, u), D(a, S a), D(u, T u), D(a, T u), D(u, S a)\} \\
& \leq r . \Phi\{d(a, u), D(a, S a), d(u, a), 0, D(u, S a)\} .
\end{aligned}
$$

If $D(S a, a) \geq d(a, u)$, it leads to contradiction. Hence $D(S a, a)<d(a, u)$. Thus

$$
D(S a, a) \leq H(S a, T u) \leq r . \Phi\{d(a, u), d(a, u), d(u, a), 0,2 d(a, u)\}
$$

That is

$$
\begin{aligned}
& D(S a, a) \leq H(S a, T u) \leq r d(a, u)<d(a, u) \Rightarrow D(u, S a)) \leq r d(a, u), \\
& D(u, T u) \leq D(u, S a)+H(S a, T u) \leq 2 r d(a, u)<D(u, T u) .
\end{aligned}
$$

This implies that $D(u, T u)=0$, that is, $u \in T u$. In similar fashion, we can show that $u \in S u$

Case 2. $u \in S u$ and $u \notin T u$ and Case 3. $u \notin S u$ and $u \in T u$ can be similarly disposed of.

Case 4 . Let $\frac{1}{2} \leq r<1$.

First we prove that

$$
H(S x, T u) \leq r \Phi\{d(x, u), D(x, S x), D(u, T u), D(x, T u), D(u, S x)\} .
$$

Assume that $x \neq u$. The for every $n \in N$, there exists $z_{n} \in S x$ such that $d\left(u, z_{n}\right) \leq D(u, S x)+\frac{1}{n} d(x, u)$. Therefore

$$
\begin{aligned}
& D(x, S x) \leq d\left(x, z_{n}\right) \leq d(x, u)+d\left(u, z_{n}\right) \\
\leq & d(x, u)+D(u, S x)+\frac{1}{n} d(x, u) \leq d(x, u)+r \cdot \max \{d(u, x), D(x, S x)\}+\frac{1}{n} d(x, u) .
\end{aligned}
$$

If $d(u, x) \geq D(x, S x)$, then we have $D(x, S x) \leq\left(1+r+\frac{1}{n}\right) d(x, u)$. Taking limit as $n \rightarrow \infty$, we have $D(x, S x) \leq(1+r) d(x, u)$. This leads to $\phi(r) D(x, S x) \leq$ $(1-r)(1+r) d(x, u) \leq d(x, u)$.

Then $\phi(r) \min \{D(x, S x), D(u, T u))\} \leq d(x, u)$. Thus

$$
H(S x, T u) \leq r \Phi\{d(x, u), D(x, S x), D(u, T u), D(x, T u), D(u, S x)\}
$$


If $d(u, x)<D(x, S x)$, similarly proceeding we have

$$
H(S x, T u) \leq r \Phi\{d(x, u), D(x, S x), D(u, T u), D(x, T u), D(u, S x)\}
$$

Let $x=u_{2 n+1}$. Then we obtain

$$
\begin{aligned}
& \quad D\left(u_{2 n+2}, T u\right) \leq H\left(S u_{2 n+1}, T u\right) \\
& \leq \\
& r \Phi\left\{d\left(u_{2 n+1}, u\right), D\left(u_{2 n+1}, S u_{2 n+1}\right), D(u, T u), D\left(u_{2 n+1}, T u\right), D\left(u, S u_{2 n+1}\right)\right\} .
\end{aligned}
$$

Taking limit as $n \rightarrow \infty$, we have

$$
D(u, T u) \leq r \Phi\{0,0, D(u, T u), D(u, T u), 0\} \leq r D(u, T u) .
$$

This implies that $D(u, T u)=0$, that is, $u \in T u$. Similarly we can show that $u \in S u$.

Corollary 3.2. If $\Phi\left\{x_{1}, x_{2}, x_{3}, x_{4}, x_{5}\right\}=\max \left\{x_{1}, x_{2}, x_{3}, \frac{x_{4}+x_{5}}{2}\right\}$, then we get Theorem $H$ of Singh et al.[18].

Corollary 3.3. If $\Phi\left\{x_{1}, x_{2}, x_{3}, x_{4}, x_{5}\right\}=\max \left\{x_{1}, \frac{x_{2}+x_{3}}{2}, \frac{x_{4}+x_{5}}{2}\right\}$, then we get Theorem I of Singh and Mishra[17].

Theorem 3.4. Let $(X, d))$ be a complete metric space and let $S, T: X \rightarrow$ $C L(X)$. Assume that there exists $r \in[0,1)$ such that for every $x, y \in X$,

$$
\varphi(r) \min \{D(x, S x), D(y, T y)\} \leq d(x, y)
$$

implies

$$
H(S x, T y) \leq r \cdot \max \{D(x, S x), D(y, T y)\}
$$

where

$$
\varphi(r)=\left\{\begin{array}{l}
1, \quad 0 \leq r<\frac{\sqrt{5}-1}{2} \\
(1-r), \frac{\sqrt{5}-1}{2} \leq r<1
\end{array}\right.
$$

Then there exists $z \in X$ such that $z \in S z \cap T z$.

Proof. Starting with $u_{0} \in X$, define $u_{2 n} \in S u_{2 n-1}$ and $u_{2 n+1} \in T u_{2 n}$ using Lemma 2.4. In either cases, $D\left(u_{2 n-1}, S u_{2 n-1}\right) \geq D\left(u_{2 n}, T u_{2 n}\right)$ or $D\left(u_{2 n-1}\right.$, $\left.S u_{2 n-1}\right) \leq D\left(u_{2 n}, T u_{2 n}\right)$ we have $\varphi(r) \min \left\{D\left(u_{2 n-1}, S u_{2 n-1}\right), D\left(u_{2 n}, T u_{2 n}\right)\right\} \leq d\left(u_{2 n-1}, u_{2 n}\right) \Longrightarrow$ 


$$
\begin{aligned}
& H\left(S u_{2 n-1}, T u_{2 n}\right) \leq r \cdot \max \left\{D\left(u_{2 n-1}, S u_{2 n-1}\right), D\left(u_{2 n}, T u_{2 n}\right)\right\} \Longrightarrow \\
& d\left(u_{2 n}, u_{2 n+1}\right) \leq k H\left(S u_{2 n-1}, T u_{2 n}\right) \leq \beta \cdot \max \left\{D\left(u_{2 n-1}, S u_{2 n-1}\right), D\left(u_{2 n}, T u_{2 n}\right)\right\} \\
& d\left(u_{2 n}, u_{2 n+1}\right) \leq \beta \cdot \max \left\{d\left(u_{2 n-1}, u_{2 n-}\right), d\left(u_{2 n}, u_{2 n+1}\right)\right\} \Longrightarrow \\
& d\left(u_{2 n}, u_{2 n+1}\right) \leq \beta d\left(u_{2 n-1}, u_{2 n-}\right),
\end{aligned}
$$

where $\beta=k r<1$ Similarly, we have $d\left(u_{2 n+2}, u_{2 n+1}\right) \leq \beta d\left(u_{2 n+1}, u_{2 n-}\right)$. Thus $d\left(u_{n+1}, u_{n}\right) \leq \beta d\left(u_{n}, u_{n-1}\right)$. Therefore $\left\{u_{n}\right\}$ is a Cauchy sequence and let $u_{n} \rightarrow$ $u \in X$.

Next we prove that

$$
D(u, S y) \leq r D(y, S y), \quad D(u, T y) \leq r D(y, T y)
$$

for all $y \in X-\{u\}$.

Since $u_{n} \rightarrow u$, there exists $n_{0} \in N$ such that $d\left(u, u_{n}\right) \leq \frac{1}{3} d(u, y)$ for $y \neq u$ and all $n \geq n_{0}$. Then

$$
\begin{aligned}
\varphi(r) d & \left(u_{2 n-1}, S u_{2 n-1}\right) \\
& \leq d\left(u_{2 n-1}, S u_{2 n-1}\right) \leq d\left(u_{2 n-1}, u_{2 n}\right) \leq d\left(u_{2 n-1}, u\right)+d\left(u, u_{2 n}\right) \\
& \leq \frac{2}{3} d(u, y)=d(u, y)-\frac{1}{3} d(u, y) \leq d(u, y)-d\left(u_{2 n-1}, u\right) \leq d\left(u_{2 n-1}, y\right) .
\end{aligned}
$$

Now either $d\left(u_{2 n-1}, S u_{2 n-1}\right) \leq d(y, T y)$ or $d(y, T y) \leq d\left(u_{2 n-1}, S u_{2 n-1}\right)$. Therefore $\varphi(r) \min \left\{d(y, T y), d\left(u_{2 n-1}, S u_{2 n-1}\right)\right\} \leq d\left(u_{2 n-1}, y\right)$. Hence, we have

$$
d\left(u_{2 n}, T y\right) \leq H\left(S u_{2 n-1}, T y\right) \leq r \cdot \max \left\{d\left(u_{2 n-1}, S u_{2 n-1}\right), d(y, T y)\right\}
$$

As $n \rightarrow \infty$, we have $d(u, T y) \leq r d(y, T y), y \in X-\{u\}$. Similarly we prove $d(u, S y) \leq r d(y, S y), \quad y \in X-\{u\}$

Next we show that $u \in S u$ and $u \in T u$.

Let $0 \leq r<\frac{\sqrt{5}-1}{2}$. Suppose $u \notin S u$ and $u \notin T u$. Let $a \in T u$. Then $a \neq u$ and we have $D(u, T a) \leq r D(a, T a)$. on the other hand

$$
\begin{aligned}
\varphi(r) D(u, T u)=D(u, T u) \leq & d(u, a) \\
& \Longrightarrow \varphi(r) \min \{D(a, S a), D(u, T u)\} \leq d(u, a) .
\end{aligned}
$$

This implies

$$
D(a, S a) \leq H(S a, T u) \leq r \cdot \max \{D(a, S a), D(u, T u)\} \leq r D(u, T u)
$$

Also when $y=a$, we have $D(u, S a) \leq r D(a, S a)$

$$
D(u, T u) \leq D(u, S a)+H(S a, T u)
$$




$$
\begin{gathered}
\leq r D(a, S a)+r \cdot \max \{D(a, S a), D(u, T u)\} \\
\leq r^{2} D(u, T u)+r D(u, T u)<D(u, T u)
\end{gathered}
$$

since $r^{2}+r<1$. Hence we obtain $u \in T u$. Similarly we can show that $u \in S u$.

Consider the case $\frac{\sqrt{5}-1}{2} \leq r<1$.

We first prove that

$$
H(S x, T u) \leq r \cdot \max \{D(x, S x), D(u, T u)\}
$$

$x \neq u$. Then for every $n \in N$, there exists $z_{n} \in S x$ such that $d\left(u, z_{n}\right) \leq$ $D(u, S x)+\frac{1}{n} d(x, u)$.

Therefore

$$
D(x, S x) \leq d\left(x, z_{n}\right) \leq d(x, u)+d\left(u, z_{n}\right) \leq d(x, u)+D(u, S x)+\frac{1}{n} d(x, u) .
$$

That is,

$$
(1-r) D(x, S x) \leq d(x, u)+\frac{1}{n} d(x, u)
$$

Taking limit as $n \rightarrow \infty$, we have $\phi(r) D(x, S x) \leq p d(x, u)$. Then

$$
\begin{aligned}
\varphi(r) \min \{D(x, S x), D(u, T u)\} & \leq d(x, u) \\
& \Longrightarrow H(S x, T u) \leq r \cdot \max \{D(x, S x), D(u, T u)\} .
\end{aligned}
$$

Let $x=u_{2 n-1}$. Then we have

$$
\begin{gathered}
H\left(S u_{2 n-1}, T u\right) \leq r \cdot \max \left\{D\left(u_{2 n-1}, S u_{2 n-1}\right), D(u, T u)\right\} \\
\lim _{n \rightarrow \infty} D\left(u_{2 n}, T u\right) \leq \lim _{n \rightarrow \infty} H\left(S u_{2 n-1}, T u\right) \\
\leq \lim _{n \rightarrow \infty} r \cdot \max \left\{D\left(u_{2 n-1}, S u_{2 n-1}\right), D(u, T u)\right\}, \\
\Longrightarrow D(u, T u) \leq r \cdot D(u, T u) \Rightarrow D(u, T u)=0 .
\end{gathered}
$$

Hence $u \in T u$.

Remark 3.5. The above theorem holds if $\varphi$ is replaced by $\phi$ (used in Theorem H).

Corollary 3.6. If $S=T$, we get Theorem L of Damjanovic and Doric[8]. 
Corollary 3.6 yields the following result and this is same as Corollary 2.1 of Damjanovic and Doric[8] which generalized Theorem J of Kikkawa and Suzuki [13].

Corollary 3.7. Let $(X, d))$ be a complete metric space and let $T: X \rightarrow$ $C B(X)$. Assume that there exists $r \in[0,1)$ such that for every $x, y \in X$,

$$
\varphi(r) d(x, T x) \leq d(x, y) \Longrightarrow H(T x, T y) \leq \alpha D(x, T x)+\alpha D(y, T y),
$$

where the function $\varphi$ is defined in Theorem3.4 and $r=2 \alpha$, that is, $\alpha \in\left[0, \frac{1}{2}\right)$. Then there exists $u \in X$ such that $u \in T u$.

\section{Application}

The existence and uniqueness of solutions of functional equations and system of functional equations that arise in dynamic programming have been studied using various fixed point theorems by several researchers ( Ref.: Z. Liu et al (2004), Doric and Lazovic(2011) and S. L. Singh et al (2012)). In this section, we prove the existence and uniqueness of a class of functional equation of dynamic programming using Corollary 3.4.

Let $U$ and $V$ be Banach spaces and $W \subset U$ and $D \subset V$. Let $R$ be the field of real numbers and let $B(W)$ denote the set of all bounded realvalued functions on $W$. The space $B(W)$ endowed with the metric $d_{B}(h, k)=$ $\sup _{x \in W}|h(x)-k(x)|, h, k \in B(W)$ is a complete metric space. According to Bellman and Lee, the basic form of the functional equation of dynamic programming is given as $p(x)=\sup _{y \in D} H(x, y, p(\tau(x, y))$, where $x$ and $y$ represent the state space and decision vectors, respectively, $\tau: W \times D \rightarrow W$ represents the transformation of the process and $p(x)$ represents the optimal return function with initial state $x$. We present the existence and uniqueness of a solution of the following functional equations:

$$
p_{i}(x)=\sup _{y \in D} H_{i}(x, y, p(\tau(x, y)), \quad i=1,2,
$$

where $H_{i}: W \times D \times R \rightarrow R$ are bounded functions. Let the mapping $A_{i}$ be defined by

$$
A_{i}(h(x))=\sup _{y \in D} H_{i}(x, y, h(\tau(x, y))\},
$$

$h \in B(W), x \in W, i=1,2$.

Using the same technique as in Sing et al [18] and Doric and Lazovic [10], the following theorem can be proved.

Theorem 4.1. Suppose that the following conditions hold: 
There exists $r \in[0,1)$ such that for every $(x, y) \in W \times D, h, k \in B(W)$ and $t \in W$, the inequality $\phi(r) \min \{|h(t)-A(h(t))|, h),\left|k(t)-A_{2}(k(t))\right| \leq$ $|h(t)-k(t)|$ implies

$$
\begin{array}{r}
\left|H_{1}(x, y, h(t))-H_{2}(x, y, k(t))\right| \leq r . \Phi\{\mid h(t)-k(t))|,| h(t)-A_{1}(h(t)) \mid, \\
\left.\left|k(t)-A_{2}(k(t))\right|,\left|h(t)-A_{2}(h(t))\right|, c\left|k(t)-A_{1} h(t)\right|\right\} .
\end{array}
$$

Then, the functional equations under consideration has a unique bounded solution in $B(W)$.

\section{References}

[1] M. Berinde and V. Berinde, On a general class of multi-valued weakly Picard mappings, J. Math. Anal., 326 (2007), 772-782.

[2] R. K. Bose, R. N. Mukherjee, Common fixed points of some multi-valued mappings, Tamkang J. Math, 8, No. 2 (1977), 245-249.

[3] R. K. Bose, M. K. Roychowdhury, Fixed point theorems for some generalized contractivemulti-valued mappings and fuzzy mappings, Mathemticki Vesnik, 63, 1 (2011), 7-26.

[4] R. K. Bose, Some Suzuki type fixed point theorems for generalized contractive multifunctions and applications, International journal of Pure and Applied Mathematics, 84(1), (20130), 13-27.

[5] L. B. Ciric, On a family of contractive maps and fixed points, Institut Mathematique Publications, Nouvelle Serie, 17(31), pp45-51, 1974.

[6] L. B. Ciric, Fixed points of for generalized multi-valued contractions, Matematicki vesnik, 9(24), pp 265-272, 1972

[7] H. Covitz, S. B. Nadler Jr., Multivalued contraction mappings in generalized metric spaces, Israel J. Math. 8 (1970) 5-11.

[8] B. Damjanovic and D. Doric, Multi-valued generalizations of the Kannan fixed point theorem, Filomat 25:1(2011), 125-131.

[9] S. Dhompongsa, H. Yingtaweesittikul, Fixed point for mulivalued mappings and the metric completeness, Fixed Point Theory and Applications, 200915 pages, Article ID 972395.doi:10.1155/2009/972395. 
[10] D. Doric and R. Lazovic, Some Suzuki-type fixed point theorems for generalized multivalued mappings and applications, Fixed point Theory and Applications 2011, 2011:40.

[11] T. Kamran, Multivalued $f$-weakly Picard mappings, Nonlinear Analysis 67 (2007), 2289-2296.

[12] M. Kikkawa, T. Suzuki, Three fixed point theorems for generalized contractions with constants in complete metric space, Nonlinear Analysis 69 (2008), 2942-2949.

[13] M. Kikkawa and T. Suzuki, Some similarity between contractions and Kannan mappings, Fixed Point Theory and Applications, 2008, Article ID $649749,1-8$.

[14] G. Mot, A. Petrusel, Fixed point theory for a new type of contracvtive multi-valued operators, Nonlinear Analysis 70 (2009), 3371-3377.

[15] S. B. Nadler, Mutivalued contraction mappings, Pacific J. Math. 30 (1969), 475-488.

[16] S. L. Singh and S. N. Mishra, Remarks on recent fixed point theorems, Fixed Point Theory and Applications, 2010Article ID 452905, 18 pages, doi:1155/2010/452905.

[17] S. L. Singh and S. N. Mishra, Coincidence theorems for certain classes of hybrid contractions, Fixed Point Theory and Applications, volume 2010, Article ID 898109, 14 pages,doi: 10.1155/2010/898109.

[18] S.L. Singh, S. N. Mishra, R. Chug and R. Kamal, General common fixed point theorems and Applications, Journal of Applied mathematics, Volume 2012, Article ID 902312, 14 pages, doi 10.1155/2012/902312.

[19] T. Suzuki, A generalized Banach contraction principle that charectreized metric completeness, Proceedings of the American Mathematical Society 136 (2008) 1861-1869.

[20] Liu, Z, Agarwal,RP, Kang, SM, On solvability of functional equations and system of functional equations arising in dynamic programming, J. Math. Anal. Appl. 297, 111-130(2004), doi:10.1016/j.jmaa2004.04.049. 
